\title{
Delirium in Pediatric Intensive Care Unit: Frequency, Causes, and Interventions
}

\author{
Sandeep Dhingra (B)
}

\begin{abstract}
Delirium is an acute neurologic dysfunction characterized by fluctuating alteration in awareness, attention, and cognition and is a result of an underlying medical condition or its treatment. It is associated with increased morbidity and mortality in the intensive care setting and may lead to long-term cognitive impairment in survivors. Pediatric delirium (PD) occurs in as many as $25 \%$ of critically ill children. The pathophysiology of delirium is complex and widespread screening of this entity is lacking. To diagnose PD, a high index of suspicion should be kept in the patients who are at high risk, and appropriate screening tools should be applied, as the symptoms of PD overlap with other commonly observed neurological phenomena in pediatric intensive care unit.
\end{abstract}

Keywords: Delirium, Pediatric intensive care unit, Pyschobehavioral interventions.

Indian Journal of Critical Care Medicine (2021): 10.5005/jp-journals-10071-23864

Delirium is an acute neurologic dysfunction characterized by fluctuating alteration in awareness, attention, and cognition and is a result of an underlying medical condition or its treatment. It is associated with increased morbidity and mortality in the intensive care setting and may lead to long-term cognitive impairment in survivors. ${ }^{1}$ Pediatric delirium (PD) occurs in as many as $25 \%$ of critically ill children. The pathophysiology of delirium is complex, and widespread screening of this entity is lacking. To diagnose PD, a high index of suspicion should be kept in the patients who are at high risk, and appropriate screening tools should be applied, as the symptoms of PD overlap with other commonly observed neurological phenomena in pediatric intensive care unit (PICU).

Delirium is independently predicted by age, developmental delay, severity of illness, coma status, mechanical ventilation (MV), use of restraints, and various medications..$^{2-4}$ It also predicts PICU length of stay (LOS), hospital LOS, duration of MV, mortality, posttraumatic stress disorder, delusional memories, and hallucinations. Incidence of delirium in ICU setting varies between $17 \%$ and $25 \%$ with a mean total duration of 2 days, ${ }^{2,4}$ while $50 \%$ of children on MV can develop delirium. ${ }^{5}$ Hyperactive delirium is the most common (48\%) while hypoactive delirium can be seen in $8 \%$ of patients. Mixed variety constitutes around $46 \%$ of cases.

Many measures have been advocated for the prevention of delirium, which includes reduced medication exposure, analgesia before sedation, avoiding benzodiazepines and anticholinergics, sedation holidays, spontaneous awake trials, and interventions to promote and protect sleep by cluster cares, soothing music and dimming lights, earplugs and eye masks, and noise reduction. Treatment of delirium revolves around looking for underlying illness, iatrogenic causes, environmental and pharmacological exposures and dealing with them.

Three validated screening tools have been developed for use in the PICU: the Pediatric Confusion Assessment Method for the ICU (pCAM-ICU), the Preschool Confusion Assessment Method for the ICU (psCAM-ICU), and the Cornell Assessment of Pediatric Delirium (CAPD). The pCAM-ICU is an interactive, cognitively oriented tool designed for children over the age 5 years, ${ }^{6}$ while the psCAM-ICU is an interactive tool used in children between 6 months
Department of Pediatrics, Command Hospital, Panchkula, Haryana, India

Corresponding Author: Sandeep Dhingra, Department of Pediatrics, Command Hospital, Panchkula, Haryana, India, Phone: +91 7030495955, e-mail: dhingrasandeep@yahoo.co.in

How to cite this article: Dhingra S, Delirium in Pediatric Intensive Care Unit: Frequency, Causes, and Interventions. Indian J Crit Care Med 2021;25(6):620-621.

Source of support: Nil

Conflict of interest: None

and 5 years of age. ${ }^{7}$ Neither is validated for use in children with developmental delay.

The CAPD is a user-friendly validated observational tool with excellent sensitivity and specificity to detect fluctuations through a continuous 8-12 hours assessment, designed for children of all ages and developmental abilities. ${ }^{8}$ CAPD should be done at least 3-4 times per day (once per shift) and a score of $\geq 9$ is taken as a positive screen. All these screening tools can be used by the bedside provider, allowing for rapid, real-time delirium screening in PICUs. The European Society of Paediatric and Neonatal Intensive Care has recommended the use of CAPD as an instrument to assess pediatric delirium in critically ill infants and children. ${ }^{9}$

The article in the current issue of IJCCM by Yöntem et al. ${ }^{10}$ has prospectively studied the frequency and causes of delirium in PICU. The authors have also evaluated the effect of psychosocial interventions on the prevalence of delirium. Intensive care environmental interventions, strategies to promote sleep, day and night orientation, and communication with the affected child have been implemented in the current study, as were advocated by Bettencourt et al. ${ }^{11}$

Results of their cohort revealed that 14 (9.9\%) children developed delirium in the PICU out of which 8 (57.1\%) had hyperactive delirium, $4(28.6 \%)$ had mixed while $2(14.3 \%)$ had hypoactive delirium. Prior single-center studies ${ }^{2,5,7,8}$ have reported PD rates ranging from 10 to $30 \%$ with many modifiable and nonmodifiable risk factors 
associated with PD. In the present study, psychosocial interventions were implemented for $76.1 \%$ of the patients which may have led to the decreased incidence of PD, which by far is the least noted in any of the prevalence studies. Though this is a single-center study with limited number of patients (142), the effect of psychosocial interventions cannot be overlooked. Larger studies employing multiple centers may give a true picture of the benefit of such interventions.

In another study, Simone et al. ${ }^{12}$ conducted a prospective study in PICU wherein delirium monitoring tool was implemented with set protocols for sedation and early mobilization. The frequency of delirium was observed to decrease from 19.3 to $11.8 \%$ postimplementation of the protocols, reiterating the benefit of such measures in reducing the incidence of delirium.

The authors noted that PD was associated with the use of respiratory support, sedation-analgesia, vasoactive and corticosteroid treatments, and physical restraints in univariate analysis. Growth and developmental delay, antibiotic use, and surgical procedures did not have any effect on delirium development. Using multivariable logistic regression adjusting for the admission PIM-2 score, presence of delirium was independently associated with psychosocial interventions and LOS in the PICU. Other prospective single-center studies of PD have demonstrated association between delirium and age less than 2 years, severity of illness, need for MV, exposure to vasopressor medications, antiepileptics, and pharmacologic sedation. ${ }^{2,5}$ Moreover, use of benzodiazepines, narcotics, and physical restraints were also strongly associated with delirium.

This study demonstrates the practicality of bedside screening of PD using CAPD and endorses the need for frequent monitoring for presence and severity of delirium in PICU. Psychosocial interventions used in this study were found to reduce the likelihood of delirium by 4.22 times, while interventions advocated by other researchers are sleep promotion, sedation, familiarity of caregivers and staff, maintenance of diurnal cycle, minimizing noise and reassurance, apart from pharmacological therapies. ${ }^{13,14}$ The authors have recognized some limitations, most notable being lack of data for prevalence of PD before implementation of psychosocial interventions; therefore, it is difficult to evaluate the exact effect of the interventions implemented. It is significant to see that the assessment of iatrogenic withdrawal syndrome was not mentioned, a disease closely related to delirium that shows similar characteristics and is one of the main differential diagnoses of delirium.

With paucity of data analyzing the effect of multicomponent nonpharmacological and pharmacological interventions, adequately powered outcome studies are needed to bridge the knowledge gap.

\section{OrCID}

Sandeep Dhingra @ https://orcid.org/0000-0002-2993-5112

\section{References}

1. Girard TD, Jackson JC, Pandharipande PP, Pun BT, Thompson $\mathrm{JL}$, Shintani AK, et al. Delirium as a predictor of long-term cognitive impairment in survivors of critical illness. Crit Care Med 2010;38(7):1513-1520. DOI: 10.1097/CCM.0b013e3181e47be1.

2. Silver G, Traube C, Gerber LM, Sun X, Kearney J, Patel A, et al. Pediatric delirium and associated risk factors: a single-center prospective observational study. Pediatr Crit Care Med 2015;16(4):303-309. DOI: 10.1097/PCC.0000000000000356.

3. Schieveld JN, Lousberg R, Berghmans E, Smeets I, Leroy PL, Vos GD, et al. Pediatric illness severity measures predict delirium in a pediatric intensive care unit. Crit Care Med 2008;36(6):1933-1936. DOI: 10.1097/ CCM.0b013e31817cee5d.

4. Smith HA, Brink E, Fuchs DC, Ely EW, Pandharipande PP. Pediatric delirium: monitoring and management in the pediatric intensive care unit. Pediatr Clin North Am 2013;60(3):741-760. DOI: 10.1016/j. pcl.2013.02.010.

5. Traube C, Silver G, Reeder RW, Doyle H, Hegel E, Wolfe HA, et al. Delirium in critically ill children: an international point prevalence study. Crit Care Med 2017;45(4):584-590. DOI: 10.1097/ CCM.0000000000002250.

6. Smith HA, Boyd J, Fuchs DC, Melvin K, Berry P, Shintani A, et al. Diagnosing delirium in critically ill children: validity and reliability of the pediatric confusion assessment method for the intensive care unit. Crit Care Med 2011;39(1):150-157. DOI: 10.1097/ CCM.0b013e3181feb489.

7. Smith HA, Gangopadhyay M, Goben CM, Jacobowski NL, Chestnut $\mathrm{MH}$, Savage $\mathrm{S}$, et al. The preschool confusion assessment method for the ICU: valid and reliable delirium monitoring for critically Ill Infants and children. Crit Care Med 2016;44(3):592-600. DOI: 10.1097/ CCM.0000000000001428.

8. Traube C, Silver G, Kearney J, Patel A, Atkinson TM, Yoon MJ, et al. Cornell assessment of pediatric delirium: a valid, rapid, observational tool for screening delirium in the PICU*. Crit Care Med 2014;42(3):656663. DOI: 10.1097/CCM.0b013e3182a66b76.

9. Harris J, Ramelet AS, van Dijk M, Pokorna P, Wielenga J, Tume L, et al. Clinical recommendations for pain, sedation, withdrawal and delirium assessment in critically ill infants and children: an ESPNIC position statement for healthcare professionals. Intensive Care Med 2016;42(6):972-986. DOI: 10.1007/s00134-016-4344-1.

10. Yontem A, Yildizdas D, Horoz O O, et al. Frequency and Causes of Delirium in Pediatric Intensive Care Unit: A Prospective Observational Study. Indian J Crit Care Med 2021;25(6):714-718.

11. Bettencourt A, Mullen JE. Delirium in children: identification, prevention, and management. Crit Care Nurse 2017;37(3):9-18. DOI: $10.4037 / \mathrm{ccn} 2017692$.

12. Simone S, Edwards S, Lardieri A, Walker LK, Graciano AL, Kishk OA et al. Implementation of an ICU bundle: an interprofessional quality improvement project to enhance delirium management and monitor delirium prevalence in a single PICU. Pediatr Crit Care Med 2017;18(6):531-540. DOI: 10.1097/PCC.0000000000001127.

13. Turkel SB. Pediatric delirium: recognition, management, and outcome. Curr Psychiatry Rep 2017;19(12):101. DOI: 10.1007/s11920-017-0851-1.

14. Kudchadkar SR, Yaster M, Punjabi NM. Sedation, sleep promotion, and delirium screening practices in the care of mechanically ventilated children: a wake-up call for the pediatric critical care community. Crit Care Med 2014;42(7):1592-1600. DOI: 10.1097/ CCM.0000000000000326. 